\title{
Analytical Study of Brain MRI Protocols, SEQUENCES AND PARAMETERS For Detection OF ABNORMALITIES
}

\author{
Shivang M. Patel ${ }^{1}$, S. M. Shah ${ }^{2}$, Priyanka Sharma ${ }^{3}$ \\ ${ }^{1}$ Department of Computer Science - IIC, Ganpat University, Ahmedabad \\ ${ }^{2}$ S. V. Institute of Computer Studies, Kadi University, Kadi \\ ${ }^{3}$ Department of Information Technology, Raksha Shakti University, Ahmedabad
}

\begin{abstract}
Image analysis by segmentation techniques has wide range of utilities in medical science like surgical planning, post- surgical assessment, abnormality detection etc. This paper is focused on various approaches for analysing the abnormalities in brain MR Images by various Brain MRI protocols, sequences and parameters used in numerous segmentation techniques like classification, clustering etc. The use of appropriate protocols, sequences and parameters set up by the referring imaging centre will prevent repeat MR scanning as well as reduce scanner time. Apart from this, current study leads to plan precise treatment for the medical practitioners/radiographers to improve accuracy and precision to detect abnormal tissues.
\end{abstract}

\section{KEYWORDS}

Segmentation, Brain MRI, Protocols, Sequences

\section{INTRODUCTION}

Detection of abnormalities from MRI images is one of the most challenging task in medical imaging research as the complexity in the anatomical structure of brain, variation in size, location and other factors like age, symptoms of patient etc. MR Images are used to produce and analyse images of soft tissue of human body without the need of surgery. Such detection requires brain image segmentation, which is the process of partitioning the image into distinct regions. The use of imaging protocols allows the standardization of procedures and workflow as well as consistency of image quality.

\section{RATIONAL OF STUDY}

There are four basic steps involved in getting an MR image. 1. Placing the patient in the magnet 2. Sending Radiofrequency (RF) pulse by coil 3. Receiving signals from the patient by coil 4. Transformation of signals into image by complex processing in the computers. [1]

MRI system is made up of several components. Any change causes potential effect in imaging. In order to reduce effects like tissue heating, muscle contractions, cardiac arrhythmias etc.; precautions requires like keep $\mathrm{dB} / \mathrm{dT}$ less than those required to produce peripheral nerve stimulation, max limit for $\mathrm{dB} / \mathrm{dT}$ of 6 Tesla / sec. [2]

DOI : $10.5121 /$ ijist.2016.6224 
MRI of the brain at 3.0 T provides a higher signal to noise ratio and allows improved spatial resolution (thinner slices, higher matrix). MRI of the brain at $3 \mathrm{~T}$ is superior in detection and accurate characterization of structural brain lesions. Compared with 1.5 T MR images, whole brain $3 \mathrm{~T}$ images are of better quality, can be performed with thinner slices, and offer superior SNR. [5]

\subsection{Image Contrast}

Images in MRI obtain contrast mainly through the processes of $\mathrm{T} 1$ recovery, $\mathrm{T} 2$ decay and proton density.

\subsubsection{T1 recovery:}

Different tissues have different $\mathrm{T} 1$ times. The $\mathrm{T} 1$ time of a tissue refers to how long that tissue takes to recover back to the longitudinal plane after an RF pulse. T1 recovery is a result of spin lattice interactions. Fat based tissues have short T1 times meaning that they give up the energy absorbed via the RF pulse quickly and thus realign with the longitudinal plane in a short amount of time. This is based on the fact that fat is hydrogen bonded to carbon and molecular mobility is restricted allowing for more efficient spin lattice interactions.

Fluids on the other hand are quite the opposite. Fluids have long T1 times meaning that they take longer to give up the energy absorbed via the RF pulse and thus take longer to realign with the longitudinal plane. Unlike fat based tissue, fluids tend to be hydrogen bonded to oxygen and molecular mobility is higher resulting in spin lattice interactions being less efficient.

In between the two extremes are water based tissues like muscles. These tissues have an intermediate $\mathrm{T} 1$ time. [3]

\subsubsection{T2 decay:}

Different tissues have different $\mathrm{T} 2$ times. The $\mathrm{T} 2$ time of a tissue refers to the amount of time it takes for the transverse magnetisation to decay. T2 decay is a result of spin spin interactions meaning the interactions of the small magnetic fields between protons.

Fat based tissues have short T2 times meaning that transverse magnetisation in fat based tissues decays rapidly. This rapid decay is again the result of the bonds between hydrogen and carbon in fat.

Fluids again are the opposite. Fluids have long T2 times meaning that after excitation by an RF pulse fluids hold there transverse magnetisation for longer times. Water based tissues such as muscle, tend to have longer T2 times than fatty based tissue however this is not always the case.

Finally it is important to note that $\mathrm{T} 1$ times of tissue are always longer than the $\mathrm{T} 2$ times. Also $\mathrm{T} 1$ and $\mathrm{T} 2$ processes are independent of one another. [3]

\subsubsection{PD of a tissue:}

It refers to the number of protons per unit volume of a particular tissue. Proton density contrast is a consequence of the relative number of protons per unit volume a tissue contains. To produce contrast differences as a result of PD the transverse component of magnetisation must reflect these differences. Tissues with a high PD will return a high signal, whereas tissues with a low PD will return a low signal. 
Different tissues have different properties however to simplify things the vast majority of tissues in the body can be broken down into three general types.

- These are fluids - eg CSF, synovial fluid and oedema.

- Water based tissues - eg muscles, brain and cartilage

- Fat based tissues -fat and bone marrow [3]

\subsection{Image Weighting}

To demonstrate either T1, PD or T2 contrast specific values of TR and TE are selected for a given pulse sequence. The selection of appropriate TR and TE weights an image so that one contrast mechanism predominates over the other two.

\subsubsection{T1 Weighting}

A T1 weighted image is one where the contrast depends on the T1 time differences between the tissues in the region on interest. TR controls how long the NMV's of the different tissues are allowed to relax back towards the longitudinal plane before being excited by the next RF pulse. To produce contrast the TR must be short enough such that none of the tissues have had time to completely relax back to the longitudinal plane. If the TR is too long then all tissues will have completely relaxed back to the longitudinal plane and upon the next RF pulse there will be no difference in the degree of transverse magnetisation and thus no signal differences to produce contrast.

At the same time a short TE is selected to minimise contrast produced as a result of T2 decay. By selecting a short TE we ensure that minimal differences in signal between tissue types will be present in the transverse plane as a result of the $\mathrm{T} 2$ decay process.

- For T1 weighting the TR must be short

- For T1 weighting the TE must be short

\subsubsection{T2 Weighting}

A T2 weighted image is one in which contrast is a result of the differences in the T2 times of the tissues being imaged. TE controls to what degree we allow the transverse magnetisation of a tissue to decay before the signal is read. To acquire T2 weighted images the TE must be long enough to give the tissues being imaged time to decay and thus allow us to exploit the differences in their T2 times. If it is too short there will not be enough signal difference between tissues and thus poor contrast.

At the same time a long TR is selected as this minimises contrast produced as a result of T1 relaxation. Using a long TR ensures full longitudinal relaxation minimising signal differences due to $\mathrm{T} 1$ relaxation properties between tissues. [3]

- For T2 weighting the TE must be long

- For T2 weighting the TR must be long

\subsubsection{PD weighting}

A PD image is one where the difference in the number of protons per unit volume of tissue is the main factor in forming image contrast. Proton density is always present to some extent, however to maximise the degree of PD weighting the effects of T1 and T2 contrast need to be minimised. 
International Journal of Information Sciences and Techniques (IJIST) Vol.6, No.1/2, March 2016

This is achieved by using a short TE to minimise contrast due to T2 decay effects and a long TR to minimise contrast as a result of $\mathrm{T} 1$ recovery.

- For PD weighting the TE must be short

- For PD weighting the TR must be long [3]

\subsection{Pre-processing}

\section{a) Size Standardization:}

The variations in the brain size and shape need to be taken into consideration before any general technique is applied.

\section{Why Image Standardization?}

- Minor differences in hardware or sequence timing i.e. TE/TR can result in significant changes in image contrast

- Time between injection and imaging affects contrast enhancement

- Difficulty in defining the exact margins and identifying the largest diameter or perpendicular diameter

For that, each MRI image is first required to crop by detecting the brain boundary and then resized to $512 \times 512$.

\section{b) Contrast Equalization:}

Variations in the MR images taken from different centers due to change in contrast level. So it is necessary to normalize all the images by iteratively increasing or decreasing the average global intensity of the image by a small amount, till we get predefined contrast level.

\section{c) Division into Active Cells:}

An $8 \times 8$ grid is placed on the image creating cells of size $64 \times 64$. The cells which do not contain any portion of brain or are partly filled are removed from consideration. These active cells are then numbered symmetrically about the vertical axis of the brain. This has been done to promote local enhancement of asymmetric regions. [4]

\subsection{Protocol, sequences \& parameters for MRI}

An MRI sequence is an ordered combination of RF and gradient pulses designed to acquire the data to form the image. The data to create an MR image is obtained in a series of steps.

First the tissue magnetisation is excited using an RF pulse in the presence of a slice select gradient.

The other two essential elements of the sequence are phase encoding and frequency encoding/read out, which are required to spatially localise the protons in the other two dimensions.

Finally, after the data has been collected, the process is repeated for a series of phase encoding steps.

The MRI sequence parameters are chosen to best suit the particular clinical application.

Imaging protocols for the brain should meet several criteria:

- They must be complete and provide all the required information 
International Journal of Information Sciences and Techniques (IJIST) Vol.6, No.1/2, March 2016

- They must be as short as possible (to minimize the time the patient has to spend in the magnet and optimize patient throughput)

- They must be reproducible

Protocols should be standardized to ensure continuity over time. Frequent changes in imaging protocols should be avoided, since this may confuse the technologists operating the MRI equipment. Obviously, imaging protocols should be adapted to the equipment available.

Some commonly used MRI sequences: [1][8]

\subsubsection{Gradient Echo Sequence:}

The gradient echo (GE) sequence is the simplest type of MRI sequence. It consists of a series of excitation pulses, each separated by a repetition time TR. Data is acquired at some characteristic time after the application of the excitation pulses and this is defined as the echo time TE. TE is the time between the mid-point of the excitation pulse and the mid-point of the data acquisition. The contrast in the image will vary with changes to both TR and TE.

In terms of k-space\# representation, the simultaneous application of the phase encode and read dephase gradients results in translation from the centre of $\mathrm{k}$-space from A to B. This is followed by frequency encoding from B to C via the centre of k-space. Each line of data is FT to extract frequency information from the signal and the process is repeated for different phase encode gradient strengths.

\#[K-Space is simply an array of numbers whose Fourier Transformation is the MR image. Simply $\mathrm{K}$-Space is best thought of as temporary image space, which is used to digitize MR signals during acquisition.]

\subsubsection{Spin Echo Sequence}

The spin echo (SE) sequence is similar to the GE sequence with the exception that there is an additional $180^{\circ}$ refocusing pulse present. This $180^{\circ}$ pulse is exactly halfway between the excitation pulse and the echo.

Due to $\mathrm{T} 2 *$ dephasing, some spins slow down and others speed up. A $180^{\circ}$ pulse is then applied to flip - the spin vectors so that the previously slower vectors are effectively processing ahead. After a further time delay (equal to TE/2), a spin echo is formed.

In terms of k-space representation of the spin-echo sequence, the application of phase encoding and read dephase gradients results in movement from the centre of k-space A to position B. The $180^{\circ}$ pulse reverses the $\mathrm{k}$-space position in both phase and frequency directions, resulting in movement from $\mathrm{B}$ to $\mathrm{C}$. This is followed by frequency encoding from $\mathrm{C}$ to $\mathrm{D}$ via the centre of $\mathrm{k}$ space (see figure 5-4). Each line of data is Fourier transformed to extract frequency information from the signal and the process is repeated for different phase encode steps.

\subsubsection{Inversion Recovery Sequence}

Inversion recovery is usually a variant of a SE sequence in that it begins with a $180^{\circ}$ inverting pulse. This inverts the longitudinal magnetisation vector through $180^{\circ}$. When the inverting pulse is removed, the magnetisation vector begins to relax back.

A $90^{\circ}$ excitation pulse is then applied after a time from the $180^{\circ}$ inverting pulse known as the TI (time to inversion). The contrast of the resultant image depends primarily on the length of the TI as well as the TR and TE. The contrast in the image primarily depends on the magnitude of the longitudinal magnetisation (as in spin echo) following the chosen delay time TI. 
International Journal of Information Sciences and Techniques (IJIST) Vol.6, No.1/2, March 2016

\subsubsection{Sagittal T1-WI}

Look for the signal intensity difference between adenohypophysis (isointense to white matter) and neurohypophysis (hyperintense).

\section{Coronal T1-WI}

Look for adenomas, which in the early phase, enhance less intensely than normal pituitary tissue.

\section{STIR (Short TI inversion Recovery)}

STIR is an inversion recovery pulse sequence that uses a TI that corresponds to the time it takes fat to recover from full inversion to the transverse plane so that there is no longitudinal magnetisation corresponding to fat. When the $90^{\circ}$ excitation pulse is applied after the delay time TI, the signal from fat is nullified. STIR is used to achieve suppression of the fat signal in a T1 weighted image. A TI of $150-175 \mathrm{~ms}$ achieves fat suppression although this value varies at different field strengths.

\section{FLAIR (FLuid Attenuated Inversion Recovery)}

FLAIR is another variation of the inversion recovery sequence. In FLAIR, the signal from fluid e.g. cerebrospinal fluid (CSF) is nulled by selecting a TI corresponding to the time of recovery of CSF from $180^{\circ}$ inversion to the transverse plane. The signal from CSF is nullified and FLAIR is used to suppress the high CSF signal in T2 and proton density weighted images so that pathology adjacent to the CSF is seen more clearly. A TI of approximately $2000 \mathrm{~ms}$ achieves CSF suppression at $1.5 \mathrm{~T}$.

\section{Fast (Turbo) Spin Echo}

With each TR in a conventional spin echo, we have a single phase-encoding step. Each of the echoes following each $180^{\circ}$ pulse is obtained after a single application of the phase-encoding gradient in conventional spin echo. Each echo has its own k-space, and each time we get an echo, we fill in one line of k-space. Fast Spin Echo is a good way of manipulating conventional spin echo to save time.

\section{Gradient Echo - Incoherent (SPGR, FLASH)}

The use of short TR intervals creates the steady state condition, but with these sequences the residual transverse magnetisation is deliberately removed or spoiled before the next excitation pulse. Spoiling is done by:

- Gradient spoiling, where a gradient of varying amplitude is turned on just before the next RF pulse.

- RF spoiling, where the phase of the RF is changed with each excitation and the receiver locks on only to the phase of the preceding pulse.

These sequences are used to produce rapid T1 weighted images. Gradient echo incoherent T1 weighted images utilise sequences with a variety of names depending upon the scanner you are using

FLASH : FAST LOW ANGLE SHOT (Siemens),

SPGR: SPOILED GRADIENT RECALLED ECHO (GE), T1-FFE : T1 FAST FIELD ECHO (Phillips), RF FAST : RF SPOILED FOURIER ACQUIRED

STEADY STATE TECHNIQUE [6] 
International Journal of Information Sciences and Techniques (IJIST) Vol.6, No.1/2, March 2016

\subsubsection{Abnormality Detection}

Before identifying abnormalities like tumor, edema, etc., it is necessary to detect regions in the T2 sequence which have properties deviating from a normal, healthy brain. Following general steps are required: [4]

1. Subtraction: $T 2$ MR image would have a hyper intense region at the location of Edema and Tumor. Using this, the histogram of each cell is obtained and subtracted with the corresponding cell of the other half. Then the abnormal region in each cell is highlighted if its value is greater than a particular threshold value.

2. Intensity Thresholding: Due to possible variation in the two halves even in the normal brain, vertical symmetry alone is not reliable. So, now the abnormal regions are again identified based on intensity thresholding. The threshold for each active cell is chosen using the robust estimate of the intensity of white matter, gray matter and CSF in the brain using a large dataset.

3. Fusion and Asymmetric Region Growing: The results are then combined to get the set of common active cells of abnormal regions. For each active cell in the set, we consider its 8neighbourhood, N8 and expand the region of asymmetry using the threshold value. This results give more refined and accurate area of abnormality.

4. Volume Calculation and Differential Classification: Calculation of the volume of the found tumor is done using the standards of MR Imaging (Slice Separation: 5mm; Slice Thickness: $1.5 \mathrm{~mm})$.

The tumor pixels found are then mapped to their anatomical locations in the brain. Based on this location and the age of patient, the differential classification of possible disorders is obtained. [7]

\section{SUMMARY}

This study aware about various MR sequences that are used in detection of abnormal tissues and their anatomical location by mapping pixels with the biological location in the brain. The classification of the possible disorders thus useful to radiologist for clinical analysis.

\section{ACKNOWLEDGMENT}

We wish to acknowledge our University, head and colleagues for their support in the completion of this paper. We are also very grateful to Gujarat Imaging Centre (Navrangpura, Ahmedabad) and its team for their immense support and help in building a varied dataset and providing us with indispensable knowledge on Brain Anatomy. We would also like to thank our family members for their kind cooperation and moral support. 
International Journal of Information Sciences and Techniques (IJIST) Vol.6, No.1/2, March 2016

\section{REFERENCES}

[1] Govind B. Chavhan, "MRI Made Easy (for Beginners), second edition, Jaypee Brothers Medcal Publishers (P) Ltd, ISBN 978-93-5090-270-7

[2] James Hancock, "MRI Study Guide" Australian Institute of Radiography 2010

[3] Westbrook, C. \& Kaut, C. 1998, Mcrobbie et. al. 2003 \& Woodward, Peggy. 2001

[4] Soniya Goyal, Sudhanshu Shekhar, K. K. Biswas, "Automatic Detection of Brain Abnormalities and Tumor Segmentationin MRI Sequence", CSE, IIT, New Delhi

[5] Paul M. Parizel, Luc van den Hauwe, Frank De Belder, J. Van Goethem , Caroline Venstermans , Rodrigo Salgado, Maurits Voormolen, and Wim Van Hecke "Magnetic Resonance Imaging of the Brain”, Springer-Verlag Berlin Heidelberg 2010

[6] Mcrobbie. D, Moore. A, Graves. M \& Prince. M, 2003, MRI From Picture to Proton, Cambridge University Press, UK, Cambridge

[7] Yu Sun, Bir Bhanu, Shiv Bhanu, "Automatic Symmetry-integrated Brain Injury Detection in MRI Sequences", 2009 IEEE

[8] Reimer, P.; Parizal, P.M.; Meaney, J.F.M.; Stichnoth, F. - A. (Eds), "Clinical MR Imaging - A Practical Approach" 2010, XX, 820 p., Hardcover, ISBN: 978-3-540-74501-3, Springer, http://www.springer.com/978-3-540-74501-3

\section{AUTHOR}

Shivang M. Patel - Research scholar from Ganpat University. S. M. Shah - Director, S. V. Institute of Computer Studies, Kadi University. Priyanka Sharma - Professor, Dept. of IT, Raksha Shakti University.

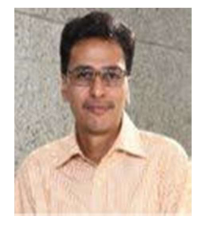

